\title{
Hedge Algorithm and Dual Averaging schemes
}

\section{Journal Article}

\section{Author(s):}

Baes, Michel; Bürgisser, Michael

Publication date:

2013-06

Permanent link:

https://doi.org/10.3929/ethz-b-000058017

Rights / license:

In Copyright - Non-Commercial Use Permitted

Originally published in:

Mathematical Methods of Operations Research 77(3), https://doi.org/10.1007/s00186-012-0418-1 


\title{
Hedge algorithm and Dual Averaging schemes
}

\author{
Michel Baes • Michael Bürgisser
}

\begin{abstract}
We show that the Hedge algorithm, a method that is widely used in Machine Learning, can be interpreted as a particular instance of Dual Averaging schemes, which have recently been introduced by Nesterov for regret minimization. Based on this interpretation, we establish three alternative methods of the Hedge algorithm: one in the form of the original method, but with optimal parameters, one that requires less a priori information, and one that is better adapted to the context of the Hedge algorithm. All our modified methods have convergence results that are better or at least as good as the performance guarantees of the vanilla method. In numerical experiments, our methods significantly outperform the original scheme.
\end{abstract}

Keywords First-order methods · Hedge algorithm · Dual Averaging methods · Convex optimization

\section{Introduction}

The Hedge algorithm was introduced by Freund and Schapire (1997) and encompasses many well-known schemes in Machine Learning. For instance, as Freund and Schapire showed, this method is related to the now widely used AdaBoost algorithm (Freund and Schapire 1997). The Hedge algorithm can be used to solve the following online allocation problem. We want to invest an amount of money in a portfolio consisting of different assets at the stock market. After each time step, we can modify the current composition of our portfolio. The Hedge algorithm defines an update strategy for our

\footnotetext{
M. Baes $(\varangle) \cdot$ M. Bürgisser

Institute for Operations Research, ETH Zürich, Rämistrasse 101, 8092 Zurich, Switzerland e-mail: michel.baes@ifor.math.ethz.ch
}

\section{Bürgisser}

e-mail: michael.buergisser@ifor.math.ethz.ch 
portfolio, such that the average performance that we achieve is not much worse than the average performance of the most favorable investment product. The portfolio update rule is based on the current loss (or gain) that is associated with every investment product.

In this paper, we propose an alternative viewpoint on the Hedge algorithm, using methods that have recently been introduced in Convex Optimization. It is well-known that the Hedge algorithm can be interpreted as a Mirror-Descent scheme (Nemirovski and Yudin 1983) with an entropy-type prox-function; see for instance Chapter 11 in Cesa-Bianchi and Lugosi (2006). However, this interpretation has two drawbacks. First, Mirror-Descent schemes require the definition of a convex and closed objective function. In this setting, the current loss of the investment products corresponds to a subgradient of this objective function. In particular, we explicitly rule out the possibility of a dynamic objective function with this approach. However, modeling the performance of a portfolio with a static objective function, even when we allow random losses, is at best questionable. As the last financial crisis has shown, significant sudden changes in the performance of an investment product can appear, which are more appropriately modeled with a dynamic objective function. Second, in order to ensure convergence, Mirror-Descent schemes need to consider subgradients with more weight the earlier they appear. However, common sense dictates that recent losses contain more relevant information on the future development of the stock market than losses occurred years ago. In this paper, we interpret the Hedge algorithm as a Dual Averaging scheme (Nesterov 2009).

Dual Averaging schemes are the natural extension of Mirror-Descent methods and get rid of both deficiencies we pointed out above at the same time. When applied to our context, Dual Averaging schemes do not make any assumptions on the construction of the losses. For instance, they can be chosen in adversarial way with respect to our current portfolio, they can be randomly generated, or-which reflects some of the latest events at the stock market more accurately - their construction rule may dynamically change. Moreover, in Dual Averaging schemes, we can give more weight to the latest losses, which allows to react much faster to significant changes in the market behavior.

Based on this alternative interpretation of the Hedge algorithm, we give three modifications of the Hedge algorithm, namely the Optimal Hedge algorithm, the Optimal Time-Independent Hedge algorithm, and the Optimal Aggressive Hedge algorithm. All these methods have convergence results that are better or at least as good as the convergence guarantee for the vanilla Hedge algorithm. The Optimal Hedge algorithm has the same form as the original Hedge algorithm, except that all method parameters are chosen in an optimal way. The Optimal Time-Independent Hedge algorithm requires less a priori information than the Optimal Hedge algorithm. Finally, the Optimal Aggressive Hedge algorithm considers losses as more relevant the later they appear. Numerical results show that all our alternative methods perform better than the vanilla Hedge algorithm. More interestingly, using the Optimal Aggressive Hedge algorithm, we end up with an average benefit that is even better than the profit of the most favorable single investment product, provided that the losses incur shocks reverting the performance of assets. This effect would not have been possible with a static objective function. 
This paper is organized as follows. In Sects. 2 and 3, we review Dual Averaging schemes and the original Hedge algorithm. We show in Sect. 4 that the Hedge algorithm is a Dual Averaging scheme and suggest several alternative methods based on this interpretation. We conclude this paper with some numerical results in Sect. 5.

\section{Dual Averaging methods}

We give a brief review of Dual Averaging schemes, which were introduced by Nesterov (2009).

Let $Q \subset \mathbb{R}^{n}$ be a closed and convex set. We assume that we have at our disposal an oracle $\mathcal{G}$, which returns a vector $g=\mathcal{G}(x) \in \mathbb{R}^{n}$ for input $x \in Q$. We interpret the oracle output $g=\mathcal{G}(x)$ as a loss vector that is associated to $x$. The corresponding loss is defined as $\langle g, x\rangle$, where $\langle\cdot, \cdot\rangle$ denotes the standard dot product in $\mathbb{R}^{n}$. Assume now that we repeat this process. That is, for $t \in \mathbb{N}$, we choose an element $x_{t} \in Q$, call the oracle $\mathcal{G}$ with input $x_{t}$, observe the loss vector $g_{t}=\mathcal{G}\left(x_{t}\right) \in \mathbb{R}^{n}$, and update our choice of the element $x_{t+1} \in Q$. After $T$ rounds, we obtain a total averaged loss of

$$
\mathcal{L}_{T}:=\frac{1}{\sum_{k=0}^{T-1} \lambda_{k}} \sum_{t=0}^{T-1} \lambda_{t}\left\langle g_{t}, x_{t}\right\rangle,
$$

where the numbers $\lambda_{0}, \ldots, \lambda_{T-1}>0$ can be seen as a tool to weight the losses according to their appearance. We can compare $\mathcal{L}_{T}$ to the averaged loss

$$
\frac{1}{\sum_{k=0}^{T-1} \lambda_{k}} \sum_{t=0}^{T-1} \lambda_{t}\left\langle g_{t}, \bar{x}\right\rangle,
$$

where $\bar{x}$ corresponds to an element in $Q$ that turns out to be optimal in hindsight. The deviation of this two quantities is called averaged regret and denoted by $\mathcal{R}_{T}$ :

$$
\begin{aligned}
\mathcal{R}_{T} & :=\frac{1}{\sum_{k=0}^{T-1} \lambda_{k}}\left(\sum_{t=0}^{T-1} \lambda_{t}\left\langle g_{t}, x_{t}\right\rangle-\min _{x \in Q}\left\{\sum_{t=0}^{T-1} \lambda_{t}\left\langle g_{t}, x\right\rangle\right\}\right) \\
& =\frac{1}{\sum_{k=0}^{T-1} \lambda_{k}} \max _{x \in Q}\left\{\sum_{t=0}^{T-1} \lambda_{t}\left\langle g_{t}, x_{t}-x\right\rangle\right\} .
\end{aligned}
$$

If the oracle $\mathcal{G}$ is associated to a convex optimization problem of the form $\min _{x \in Q} f(x)$, that is, the oracle return correspond to subgradients of $f$, the averaged regret $\mathcal{R}_{T}$ gives us an upper bound on the optimality gap $\min _{0 \leq t \leq T-1} f\left(x_{t}\right)-\min _{x \in Q} f(x)$.

Naturally, the following question arises: is there a strategy to update the elements $x_{0}, \ldots, x_{T-1}$ such that the averaged regret $\mathcal{R}_{T}$ is bounded from above by a quantity that converges to zero when $T$ goes to infinity? Nesterov's Dual Averaging schemes (Nesterov 2009) can be used to define such update strategies. 
We equip $\mathbb{R}^{n}$ with a norm $\|\cdot\|$, not necessarily the norm associated with $\langle\cdot, \cdot\rangle$, and denote by $\|\cdot\|_{*}$ the corresponding dual norm. Nesterov's Dual Averaging methods require a prox-function $d: Q \rightarrow \mathbb{R}$, that is, a function that is continuous and strongly convex modulus $\sigma>0$ with respect to $\|\cdot\|$ on $Q$. We set $x_{0}=\arg \min _{x \in Q} d(x)$. Without loss of generality, we may assume that $\sigma=1$ and that $d$ vanishes at $x_{0}$. The algorithm accumulates all the loss vectors in a dual variable $s_{t+1}$, that is, $s_{t+1}=$ $-\sum_{k=0}^{t} \lambda_{k} g_{k}$ for any $t=0, \ldots, T-1$. In order to define $x_{t+1}$, the dual variable $s_{t+1}$ is then projected back on the set $Q$ using the parameterized mirror-operator

$$
\pi_{Q, \beta_{t+1}}: \mathbb{R}^{n} \rightarrow Q: s \mapsto \arg \max _{x \in Q}\left\{\left\langle s, x-x_{0}\right\rangle-\beta_{t+1} d(x)\right\},
$$

where $\beta_{t+1}>0$ is some projection parameter. We assume that $d$ is chosen in such a way that the above optimization problem is easily solvable. The resulting scheme looks as follows.

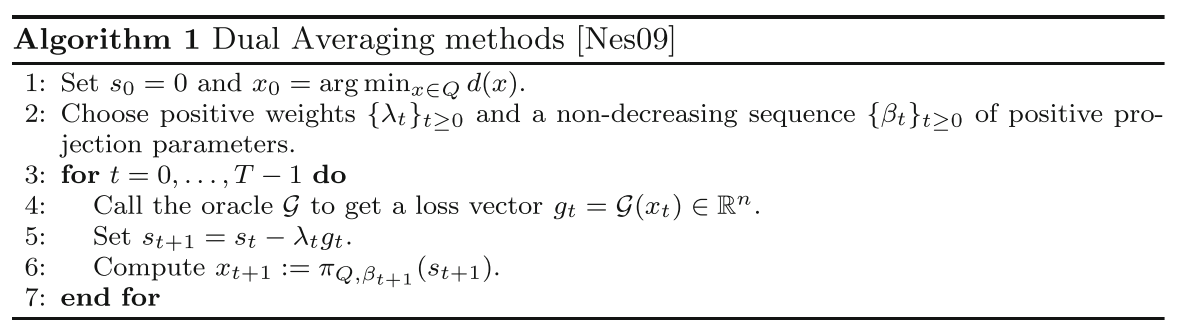

Nesterov proved the following result for this method.

Theorem 1 (First part of Theorem 1 in Nesterov 2009) For any $D \geq 0$, we have:

$$
\begin{aligned}
& \frac{1}{\sum_{k=0}^{T-1} \lambda_{k}} \max _{x \in Q}\left\{\sum_{t=0}^{T-1} \lambda_{t}\left\langle g_{t}, x_{t}-x\right\rangle: d(x) \leq D\right\} \\
& \leq \frac{1}{\sum_{k=0}^{T-1} \lambda_{k}}\left(\beta_{T} D+\frac{1}{2} \sum_{t=0}^{T-1} \frac{\lambda_{t}^{2}}{\beta_{t}}\left\|g_{t}\right\|_{*}^{2}\right)
\end{aligned}
$$

Let us assume that the oracle returns are uniformly bounded, that is, there exist a constant $L$ such that $\left\|g_{t}\right\|_{*} \leq L$ for any $t=0, \ldots, T-1$. The above theorem motivates several ways to choose the weights $\lambda_{t}$ and the projection parameters $\beta_{t}$. For instance, we can set $\beta_{t}=1$ for any $t$ and choose constant weights $\lambda_{t}=\lambda^{*}$ in such a way that the right-hand side in (1) is minimized. That is, provided that $T$ is fixed in advance, we set $\lambda^{*}=(1 / L) \sqrt{2 D / T}$, for which the right-hand side in (1) becomes $L \sqrt{2 D / T}$. Moreover, Nesterov (2009) observed that for $\beta_{t}=1$ the right-hand side in (1) converges to zero as long as as $\sum_{t=0}^{T} \lambda_{t}$ diverges and $\sum_{t=0}^{T} \lambda_{t}^{2}$ converges when $T$ goes to infinity. The latter condition implies that the weights $\lambda_{t}$ converge to zero. Selecting the $\beta_{t}$ 's in an appropriate way, we can allow non-decreasing weights $\lambda_{t}$ while still ensuring that the right-hand side in (1) converges to zero when $T$ goes to infinity. For instance, as Nesterov (2009) suggested, we can set 


$$
\lambda_{t}=\frac{\sqrt{2 D}}{L}, \quad \beta_{0}=1, \quad \text { and } \quad \beta_{t+1}=\sum_{k=0}^{t} \frac{1}{\beta_{t}} \quad \forall t \geq 0
$$

for which the right-hand side in (1) is still in $\mathcal{O}(L \sqrt{D / T})$. The same asymptotic bound can be guaranteed for $\lambda_{t}=(t+1)^{2} \sqrt{7 D} / L$ and $\beta_{t}=t^{2.5}$ for each $t \geq 0$.

\section{The Hedge algorithm}

The Hedge algorithm (Freund and Schapire 1997) is a generic method that encompasses many well-known schemes in Machine Learning. As examples, Multiplicative Weights Update methods are a variation of the Hedge algorithm (see Arora et al. 2005 for a survey) and AdaBoost can be related to the Hedge algorithm (see Freund and Schapire 1997 for more details).

The problem the Hedge algorithm aims at solving can be described as follows. We assume that we want to invest a certain amount of money at the stock market. We have at our disposal a basket of $n$ investment products such as shares, currencies, gold, raw materials, real estates, and so on. Let us denote by $x_{t, i} \geq 0$ the share of our initial amount of money that we invest in product $i$ at time $t$, where $i=1, \ldots, n$ and $t \geq 0$. We always invest all of our money, that is, we assume $\sum_{i=1}^{n} x_{t, i}=1$ for all $t \geq 0$. At every time step $t \geq 0$, we can evaluate the loss (or gain) $\ell_{t, i}$ corresponding to the investment product $i$, where we assume $\ell_{t, i} \in[-\mu, \rho]$ for every $t \geq 0$ and any $i=1, \ldots, n$. Thus, given our portfolio $x_{t}$ at time $t$, we suffer a loss of $\left\langle\ell_{t}, x_{t}\right\rangle$ at this time step. The Hedge algorithm defines now an update strategy for our portfolio such that the averaged loss $\sum_{t=0}^{T-1}\left\langle\ell_{t}, x_{t}\right\rangle / T$ that we face is not much worse than the averaged total loss $\min _{1 \leq i \leq n} \sum_{t=0}^{T-1} \ell_{t, i} / T$ of the investment product with the best performance.

The Hedge scheme evaluates the losses through a decreasing score function $U$ : $[-\mu, \rho] \rightarrow(0,1]$. For the sake of brevity, we focus in this paper only on score functions of the form $U(z)=\gamma^{a z+b}$, where $\gamma \in(0,1), a>0$, and $b \in \mathbb{R}$ are some parameters whose choices we discuss in detail afterwards. The Hedge algorithm assigns a weight $w_{t, i}$ to every investment product $1 \leq i \leq n$ and for every time step $t \geq 0$. The current weight of investment product $i$ depends on its initial weight and on its performance in the past. More concretely, it is defined as $w_{t+1, i}:=w_{t, i} U\left(\ell_{t, i}\right)$. The portfolio $x_{t+1}$ is then given by the normalization of the weight vector $w_{t+1}$. The full method takes the following form.

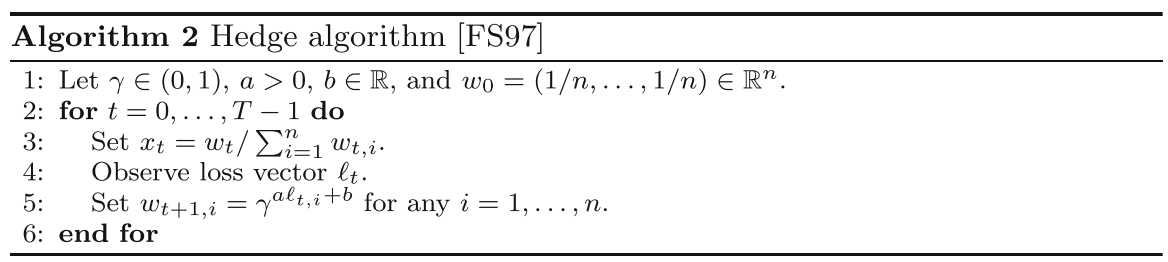

Freund and Schapire studied the convergence behavior of Algorithm 2. In their paper, they considered the situation where $\mu=0$ and $\rho=1$. The immediate extension of their reasoning to our more general setting yields to the following result. 
Theorem 2 (Extension of Theorem 2 in Freund and Schapire 1997) With $a=1 /(\mu+$ $\rho)$ and $b=\mu /(\mu+\rho)$, the sequence $\left(x_{t}\right)_{t=0}^{T-1}$ generated by Algorithm 2 satisfies

$$
\sum_{t=0}^{T-1}\left(\mu+\left\langle\ell_{t}, x_{t}\right\rangle\right) \leq \frac{\mu+\rho}{1-\gamma}-\frac{\ln (\gamma)}{1-\gamma} \min _{1 \leq i \leq n}\left(\sum_{t=0}^{T-1}\left(\mu+\ell_{t, i}\right)\right) .
$$

As mentioned in Freund and Schapire (1997), the above theorem can be extended to any decreasing score function $U:[-\mu, \rho] \rightarrow \mathbb{R}$ that complies with the condition

$$
\gamma^{\frac{z+\mu}{\mu+\rho}} \leq U(z) \leq 1-(1-z) \frac{z+\mu}{\mu+\rho} \quad \forall z \in[-\mu, \rho]
$$

In accordance with Freund and Schapire (1997), we set $\gamma=1 /(\sqrt{2 \ln (n) / T}+1)$. With this parameter setting, we obtain the score function

$$
U:[-\mu, \rho] \rightarrow \mathbb{R}: z \mapsto(\sqrt{2 \ln (n) / T}+1)^{-\frac{z+\mu}{\mu+\rho}}
$$

for which one can prove the following statement using Theorem 2; see Freund and Schapire (1997) for more details on the derivation of this score function.

Corollary 1 (Consequence of Lemma 4 in Freund and Schapire 1997) With the above score function, we have:

$$
\frac{1}{T}\left(\sum_{t=0}^{T-1}\left\langle\ell_{t}, x_{t}\right\rangle-\min _{1 \leq i \leq n} \sum_{t=0}^{T-1} \ell_{t, i}\right) \leq(\mu+\rho)\left(\frac{\ln (n)}{T}+\sqrt{\frac{2 \ln (n)}{T}}\right) .
$$

\section{The Hedge algorithm is a Dual Averaging method}

We show that we can recast the Hedge algorithm in the framework of Dual Averaging schemes and derive alternative versions of the original method.

We define $Q$ as the $(n-1)$-dimensional standard simplex

$$
\Delta_{n}=\left\{x \in \mathbb{R}^{n}: x \geq 0, \sum_{i=1}^{n} x_{i}=1\right\}
$$

so that $Q$ encompasses all possible portfolios. We equip $\mathbb{R}^{n}$ with the norm $\|x\|_{1}:=$ $\sum_{i=1}^{n}\left|x_{i}\right|$. The corresponding dual norm is of the form $\|s\|_{\infty}:=\max _{1 \leq i \leq n}\left|s_{i}\right|$. Moreover, we endow Algorithm 1 with the prox-function

$$
d_{\Delta_{n}}: \Delta_{n} \rightarrow \mathbb{R}: x \mapsto \ln (n)+\sum_{i=1}^{n} x_{i} \ln \left(x_{i}\right)
$$


It is well-known that $d_{\Delta_{n}}$ complies with our assumptions on prox-functions, that is, it is continuous and strongly convex modulus 1 with respect to $\|\cdot\|_{1}$ on $\Delta_{n}$, it attains its center $x_{0}:=\arg \min _{x \in \Delta_{n}} d_{\Delta_{n}}(x)$ at $(1 / n, \ldots, 1 / n)$, and it vanishes at this point; see for instance Nesterov (2009) and the references therein. Moreover, we can explicitly write the corresponding parameterized mirror-operator:

$$
\pi_{\Delta_{n}, \beta}(s)=\left(\frac{\exp \left(s_{i} / \beta\right)}{\sum_{j=1}^{n} \exp \left(s_{j} / \beta\right)}\right)_{i=1}^{n} \quad \forall s \in \mathbb{R}^{n}, \forall \beta>0 .
$$

Given a loss vector $\ell_{t} \in \mathbb{R}^{n}$, that is, $\ell_{t, i}$ corresponds to the loss of investment product $i$ at time $t$, we evaluate this vector through an affine function $z \mapsto a z+b$, where $a>0$ and $b \in \mathbb{R}$. This affine function can be interpreted as how we subjectively perceive a loss $z$. We use the resulting vector $g_{t}:=\left(a \ell_{t, i}+b\right)_{i=1}^{n}$ as the output $\mathcal{G}\left(x_{t}\right)$ of the oracle $\mathcal{G}$ in Algorithm 1 for input vector $x_{t} \in \mathbb{R}^{n}$,. Note that we do not specify any construction rule for the loss vector $\ell_{t}$. For instance, they could be chosen randomly or in an adversarial way with respect to the portfolio $x_{t}$. Algorithm 1 takes the following form for our setting, where we express the parameterized mirror-operator $\pi_{\Delta_{n}, \beta}$ in a form that makes the comparison of the resulting method with the Hedge algorithm rather transparent.

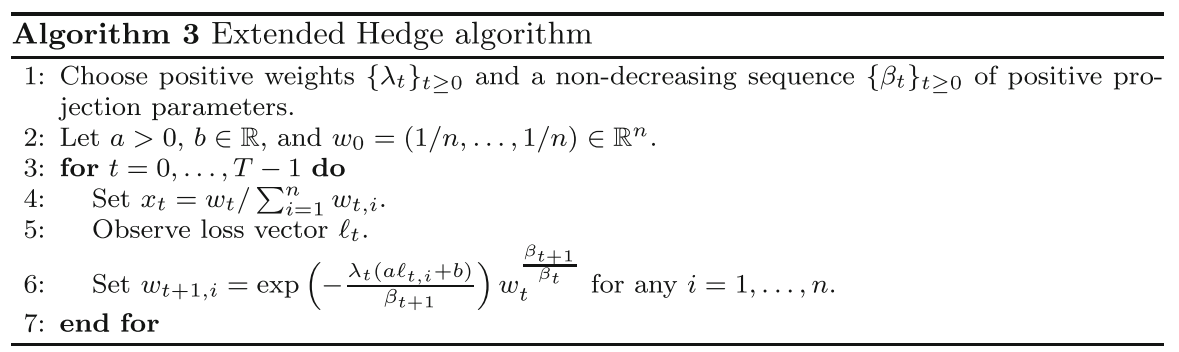

Let us now discuss several strategies for choosing the weights $\gamma_{t}$, the projection parameters $\beta_{t}$, and the affine function $z \mapsto a z+b$ in Algorithm 3. However, first we observe that the norm of each oracle return $a \ell_{t}+b$ and the prox-function $d_{\Delta_{n}}$ are bounded from above by the quantities $L(a, b):=\max \{|-a \mu+b|,|a \rho+b|\}$ and by $D:=\ln (n)$, respectively.

Original Hedge algorithm: If $\beta_{t}=1$ and $\lambda_{t}=\ln (1 / \gamma)$ for any $t=0, \ldots, T-1$ and with a fixed $\gamma \in(0,1)$, we recover the Hedge algorithm. This implies that the Hedge algorithm is Dual Averaging scheme.

Optimal Hedge algorithm: Theorem 1 yields for these weights and projection parameters:

$$
\frac{1}{T} \max _{x \in \Delta_{n}} \sum_{t=0}^{T-1}\left\langle\ell_{t}, x_{t}-x\right\rangle \leq \frac{1}{a T \ln (1 / \gamma)}\left(D+\frac{1}{2} \sum_{t=0}^{T-1} \ln ^{2}(1 / \gamma) L^{2}(a, b)\right) .
$$


This bound suggests an optimal way of selecting the parameters $b^{*}$ and $\gamma^{*}$ when $a$ is fixed to some value $a^{*}>0$. The right-hand side is indeed minimized if these numbers satisfy the relations:

$$
b^{*}=\frac{\mu-\rho}{2} a^{*} \quad \text { and } \quad \gamma^{*}=\exp \left(-\frac{2}{a^{*}(\mu+\rho)} \sqrt{\frac{2 \ln (n)}{T}}\right) .
$$

Interestingly, the particular value of the loss perception factor $a^{*}>0$ does not influence the complexity bound of the algorithm, as for every $a^{*}>0$ we have:

$$
\frac{1}{T} \max _{x \in \Delta_{n}} \sum_{t=0}^{T-1}\left\langle\ell_{t}, x_{t}-x\right\rangle \leq(\mu+\rho) \sqrt{\frac{\ln (n)}{2 T}} .
$$

We refer to Algorithm 3 with the setting specified just above as Optimal Hedge algorithm, for which This result improves Bound (5) by the additive quantity $(\mu+\rho) \ln (n) / T$ and by a multiplicative factor of 2 . Note that the resulting score function $U(z)=\left(\gamma^{*}\right)^{a^{*} z+b^{*}}$ does not comply with Condition (3). Therefore, neither Theorem 2 nor its extension can be used to establish the above bound.

Optimal Time-Independent Hedge algorithm: The update parameter $\gamma$ depends on the number of iterations $T$ in both algorithms, the Original Hedge algorithm with the score function (4) suggested by Freund and Schapire (1997) and the Optimal Hedge algorithm. However, when investing our money at the stock market, we might not want to fix the number of times that we adapt our portfolio in advance. We thus need an update parameter that is independent of $T$. Adapting Nesterov's strategy (2), we choose $\gamma \in(0,1)$ and set $\lambda_{t}=\ln (1 / \gamma), \beta_{0}=1$, and $\beta_{t+1}=\sum_{k=0}^{t-1} 1 / \beta_{k}$ for any $t \geq 0$. Applying Theorem 1 , we obtain for any $T \geq 1$ :

$$
\frac{1}{T} \max _{x \in \Delta_{n}} \sum_{t=0}^{T-1}\left\langle\ell_{t}, x_{t}-x\right\rangle \leq \frac{\beta_{T}}{a T \ln (1 / \gamma)}\left(D+\frac{1}{2} \ln ^{2}(1 / \gamma) L^{2}(a, b)\right) .
$$

We minimize the right-hand side of the above inequality, that is, we choose a loss perception factor $a^{*}>0$ and set

$$
\gamma^{*}=\exp \left(-\frac{2 \sqrt{2 \ln (n)}}{a^{*}(\mu+\rho)}\right) \quad \text { and } \quad b^{*}=\frac{\mu-\rho}{2} a^{*}
$$

Exploiting Lemma 3 in Nesterov (2009), we obtain for the resulting method, which we refer to as the Optimal Time-Independent Hedge algorithm, the following inequalities for all $T \geq 1$ : 


$$
\begin{aligned}
& \frac{1}{T} \max _{x \in \Delta_{n}} \sum_{t=0}^{T-1}\left\langle\ell_{t}, x_{t}-x\right\rangle \leq(\mu+\rho)\left(\frac{1}{(1+\sqrt{3}) T}+\sqrt{\frac{2}{T}}\right) \sqrt{\frac{\ln (n)}{2}} \\
& \quad \leq 2(\mu+\rho) \sqrt{\frac{\ln (n)}{T}} .
\end{aligned}
$$

Optimal Aggressive Hedge algorithm: The later a loss appears, the more likely it is that this loss vector contains relevant information for the future development of the investment products' performances. We conclude this section by introducing an alternative version of the Hedge algorithm, where we continuously increase the weights of the loss vectors when time proceeds. For fixed $\gamma \in(0,1)$, we set $\lambda_{t}=\ln (1 / \gamma)(t+1)^{2}$ and $\beta_{t}=t^{2.5}$ for any $t \geq 0$. Let $T>6$. Using the relations $\sum_{t=0}^{T-1}(t+1)^{2}=T(T+1)(2 T+1) / 6>T^{3} / 3, \sum_{t=0}^{T-1}(t+1)^{4} \leq 2 T^{5} / 7$, and Theorem 1, we obtain for Algorithm 3:

$$
\begin{aligned}
& \frac{6}{T(T+1)(2 T+1)} \max _{x \in \Delta_{n}} \sum_{t=0}^{T-1}(t+1)^{2}\left\langle\ell_{t}, x_{t}-x\right\rangle \\
& \quad<\frac{3}{a T^{3}}\left(\frac{T^{2.5} D}{\ln (1 / \gamma)}+\frac{1}{2} \sum_{t=0}^{T-1} \frac{(t+1)^{4} \ln (1 / \gamma)}{T^{2.5}} L^{2}(a, b)\right) \\
& \leq \frac{3}{a \sqrt{T}}\left(\frac{D}{\ln (1 / \gamma)}+\frac{\ln (1 / \gamma) L^{2}(a, b)}{7}\right) .
\end{aligned}
$$

Fixing $a$ to a constant $a^{*}>0$, the latter quantity is minimized for $b^{*}$ and $\gamma^{*}$ satisfying

$$
b^{*}=\frac{\mu-\rho}{2} a^{*} \quad \text { and } \quad \gamma^{*}=\exp \left(-\frac{2 \sqrt{7 \ln (n)}}{a^{*}(\mu+\rho)}\right)
$$

We call the resulting method Optimal Aggressive Hedge algorithm, for which we can rewrite the above inequality as:

$$
\frac{6}{T(T+1)(2 T+1)} \max _{x \in \Delta_{n}} \sum_{t=0}^{T-1}(t+1)^{2}\left\langle\ell_{t}, x_{t}-x\right\rangle<3(\mu+\rho) \sqrt{\frac{\ln (n)}{7 T}} .
$$

Note that the averaged regret reflects our time-varying choice of the weights $\lambda_{t}$.

\section{Numerical results}

We select a pool of $n=30$ investment products and consider $T=31,200$ iterations of the methods that we presented. The number $T$ is chosen in such a way that it corresponds to the number of transactions at a stock exchange during four months (20 trading days of $6 \mathrm{~h} 30$ for one month), provided that there is transaction every minute. The losses $\ell_{t} \in \mathbb{R}^{n}, t=0, \ldots, T-1$, are randomly generated. The first 7,800 


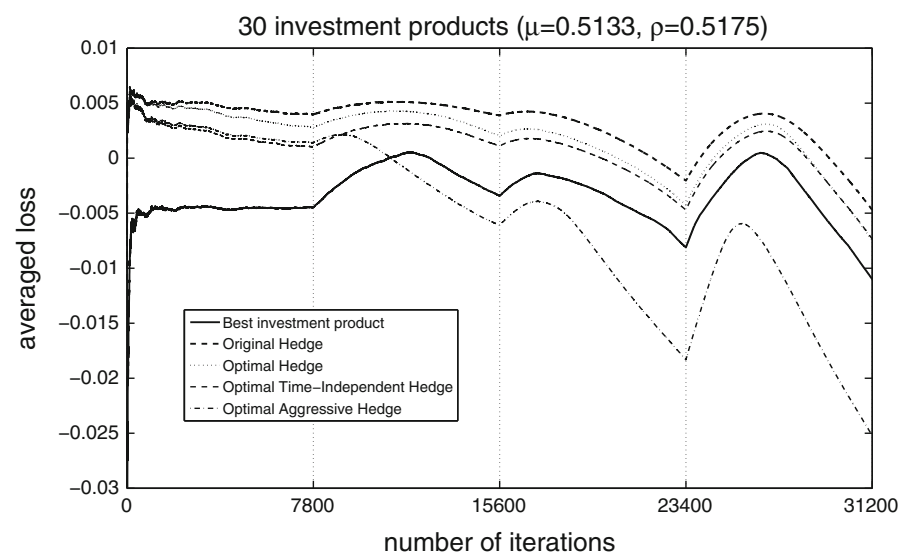

Fig. 1 Averaged losses $\sum_{k=1}^{t}\left\langle\ell_{k-1}, x_{k-1}\right\rangle / t, t=1, \ldots, T$, achieved by the best investment product (thick line), by the Original Hedge algorithm (thick dashed line), by the Optimal Hedge algorithm (dotted line), by the Optimal Time-Independent Hedge algorithm (thin dashed line), and by the Optimal Aggressive Hedge algorithm (dashed-dotted line)

Table 1 Averaged losses achieved by the best investment product, by the Original Hedge algorithm, by the Optimal Hedge algorithm, by the Optimal Time-Independent Hedge algorithm, and by the Optimal Aggressive Hedge algorithm after one, two, three, and four months of trading

\begin{tabular}{lrrrrl}
\hline Number of iterations & 7,800 & 15,600 & 23,400 & 31,200 & w.r.t. best product $(\%)$ \\
\hline 30 investment products $(\mu=0.5133, \rho=0.5175)$ & & & \\
Best investment product & -0.0045 & -0.0034 & -0.0081 & -0.0110 & - \\
Original Hedge & 0.0040 & 0.0039 & -0.0020 & -0.0047 & 42.7 \\
Optimal Hedge & 0.0028 & 0.0020 & -0.0042 & -0.0075 & 68.2 \\
Optimal Time-Independent Hedge & 0.0010 & 0.0011 & -0.0047 & -0.0073 & 66.4 \\
Optimal Aggressive Hedge & 0.0014 & -0.0061 & -0.0183 & -0.0252 & 229.1 \\
\hline
\end{tabular}

In the last column, we express the final averaged loss in percentage of the final averaged loss achieved by the best investment product

losses $\left(\ell_{t}\right)_{t=0}^{7799}$, that is, the losses observed during the first month, are realizations of a multivariate normally distributed random vector with mean $\bar{\mu}_{1}$ and covariance matrix $\Sigma$. The data $\left(\bar{\mu}_{1}, \Sigma\right)$ is taken from http://tabu.diegm.uniud.it/portfolio/. The losses $\left(\ell_{7800(j-1)+k}\right)_{k=0}^{7799}$ observed in month $j$, where $j=2,3,4$, are realizations of a multivariate normally distributed random vector with the same covariance matrix $\Sigma$, but with a different mean $\bar{\mu}_{j}$. In our experiments, we modify each component $\bar{\mu}_{j-1, i}$ of $\bar{\mu}_{j-1}$ as $\bar{\mu}_{j, i}=a_{j, i} \bar{\mu}_{j-1, i}+b_{j}$, with $b_{j}$ small. The coefficient $a_{j, i}$ is negative with an increasing probability as $j$ increases (namely $1 / 2,3 / 4$, and 1 ), reverting the performance of more and more products. The level of perturbation $\left|a_{j, i}\right|$ is also increasing as $j$ increases. The experiments are run 10 times, and the obtained losses are averaged afterwards.

In Fig. 1, we show the averaged losses, that is, $\sum_{k=1}^{t}\left\langle\ell_{k-1}, x_{k-1}\right\rangle / t$ for any $t \geq 1$, achieved by the most successful investment product at instant $t$ (obviously, this winning 
product might change over time), by the Original Hedge algorithm [with Freund and Schapire's score function as described in (4)], by the Optimal Hedge algorithm, by the Optimal Time-Independent Hedge algorithm, and by the Optimal Aggressive Hedge algorithm. Note that we show for the Optimal Aggressive Hedge algorithm also the quantity $\sum_{k=1}^{t}\left\langle\ell_{k-1}, x_{k-1}\right\rangle / t$, although we use a different weighting in the algorithm and in its theoretical analysis; compare with the last section. In Table 1, we give the averaged losses after each month.

We observe that all the extensions of the Hedge algorithm that we suggested in this paper significantly outperform its original counterpart. Even more interestingly, the Optimal Aggressive Hedge algorithm achieves an averaged loss that is more than two times better than the averaged loss of the best investment product after 4 months. The Optimal Aggressive Hedge algorithm outperforms the most successful investment product, as the investment product with the best performance has accumulated a significant loss in an early month. This happens as we switch signs when we perturb the means of the distribution that we use to generate random losses.

Compared to the other versions of the Hedge algorithm that we suggested in this paper, the Optimal Hedge algorithm reacts faster and thus more successful to the perturbations. This is due to the increasing weights $\lambda_{t}$, which makes losses the more relevant the later they appear. Recall that all the other methods consider the losses as equally important.

\section{References}

Arora S, Hazan E, Kale S (2005) Multiplicative weights method: a meta-algorithm and applications. Technical report, Computer Science Department, Princeton

Cesa-Bianchi N, Lugosi G (2006) Prediction, learning, and games, Cambridge University Press, Cambridge Freund Y, Schapire R (1997) A decision-theoretic generalization of on-line learning and an application to boosting. J Comput Syst Sci 55:119-139

Nesterov Y (2009) Primal-dual subgradient methods for convex problems. Math Program 120(1):221-259

Nemirovski A, Yudin D (1983) Problem complexity and method efficiency in optimization, Wiley. London Portfolio selection instances from Yahoo Finance. Available from http://tabu.diegm.uniud.it/portfolio/ 